\title{
Correction to: Turbulence: A Cooperative Phenomenon
}

\section{Correction to:}

\section{Chapter 11 in: P. W. Egolf, K. Hutter,}

Nonlinear, Nonlocal and Fractional Turbulence, https://doi.org/10.1007/978-3-030-26033-0_11

The original version of the book was inadvertently published with an error in Table 11.2. Owing to an oversight Table 7.2 was mistakenly duplicated as Table 11.2. The chapter has now been corrected and approved by the author. The correct presentation is included now. 
Table 11.2 Quantities of critical exponents given by models and experiments for a selection of different physical systems for the range $T<T_{\mathrm{c}}$. Data are from Ma (1982) and Stanley (1971)

\begin{tabular}{lccccc}
\hline \hline Quantity & $\alpha$ & $\beta^{\prime}$ & $\delta$ & $\gamma^{\prime}$ & $v^{\prime}$ \\
\hline Fluids & - & - & - & - & - \\
\hline $\mathrm{CO}_{2}$ & $\sim 0.1$ & 0.34 & 4.2 & $\sim 1.0$ & - \\
\hline Xe & $<0.2$ & 0.35 & 4.4 & $\sim 1.2$ & 0.57 \\
\hline Magnets & - & - & & - & - \\
\hline Ni & -0.3 & 0.42 & 4.22 & 1.32 & - \\
\hline EuS & -0.15 & 0.33 & - & - & - \\
\hline CrBr3 & - & 0.368 & 4.3 & - & - \\
\hline Models & - & - & & -- & - \\
\hline Classical & 0 & $1 / 2$ & 3 & 1 & $1 / 2$ \\
\hline Spherical 3-d & - & $1 / 2$ & 5 & - & - \\
\hline Ising 2-d & 0 & $1 / 8$ & $\sim 15$ & $\sim 7 / 4$ & 1 \\
\hline Ising 3-d & $\sim 1 / 8$ & $\sim 5 / 16$ & $\sim 5$ & $\sim 5 / 4$ & - \\
\hline Heisenberg3-d & - & $\sim 0.35$ & $\sim 5$ & - & - \\
\hline \hline
\end{tabular}

